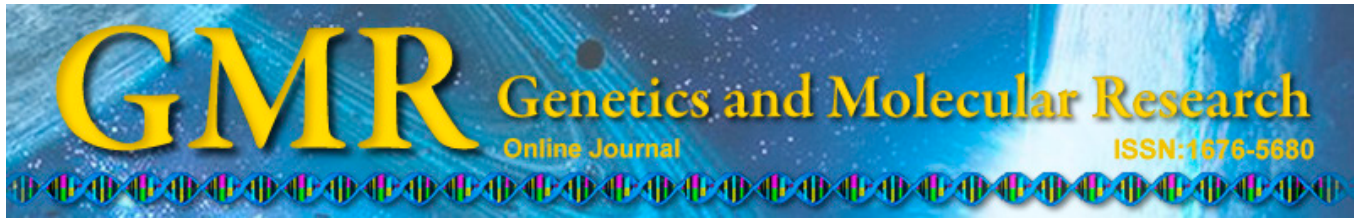

\title{
Effectiveness of external fixator combined with T-plate internal fixation for the treatment of comminuted distal radius fractures
}

\author{
L.R. Han, C.X. Jin, J. Yan, S.Z. Han, X.B. He and X.F. Yang \\ Department of Orthopaedics, Liaocheng People's Hospital, Liaocheng, China \\ Corresponding author: J. Yan \\ E-mail: junycn@yeah.net
}

Genet. Mol. Res. 14 (1): 2912-2919 (2015)

Received June 24, 2014

Accepted October 23, 2014

Published March 31, 2015

DOI http://dx.doi.org/10.4238/2015.March.31.22

\begin{abstract}
This study compared the efficacy between external fixator combined with palmar T-plate internal fixation and simple plate internal fixation for the treatment of comminuted distal radius fractures. A total of 61 patients classified as type $\mathrm{C}$ according to the $\mathrm{AO} / \mathrm{ASIF}$ classification underwent surgery for comminuted distal radius fractures. There were 54 and 7 cases of closed and open fractures, respectively. Moreover, 19 patients received an external fixator combined with T-plate internal fixation, and 42 received simple plate internal fixation. All patients were treated successfully during 12-month postoperative follow-up. The follow-up results show that the palmar flexion and dorsiflexion of the wrist, radial height, and palmar angle were significantly better in those treated with the external fixator combined with T-plate compared to those treated with the simple plate only $(\mathrm{P}<0.05)$; however, there were no significant differences in radial-ulnar deviation, wrist range of motion, or wrist function score between groups $(\mathrm{P}>0.05)$. Hence, the effectiveness of external fixator combined with T-plate internal fixation for the treatment of comminuted distal radius fractures was satisfactory. Patients sufficiently recovered wrist, forearm, and hand function.
\end{abstract}


In conclusion, compared to the simple T-plate, the external fixator combined with T-plate internal fixation can reduce the possibility of the postoperative re-shifting of broken bones and keep the distraction of fractures to maintain radial height and prevent radial shortening.

Key words: Radius; Fractures; External fixator; T-plate

\section{INTRODUCTION}

Severe comminuted distal radius fractures are unstable fractures that mostly involve the surface of the radiocarpal or distal radioulnar joint. Treatment involves resetting articular surfaces, restoring radial length, correcting ulnar deviation and palmar angle, resetting the radiocarpal or distal radioulnar joint, and maintaining the structure after reset. Inappropriate treatments can cause the degeneration of the wrist joint, pain, stiffness, and other symptoms as well as obvious changes in the articular surface of the distal radius, ulnar deviation, palmar angle, and radial shortening, which severely limit the forearm rotation and wrist function.

For distal radius fractures classified as type A or B according to the AO/ASIF classification, the application of plaster external fixation can achieve satisfactory therapeutic results (Al Khudairy et al., 2013). However, for fractures involving the articular surface and unstable comminuted fractures, it is difficult to maintain a good reset by using common external fixation. This can easily cause distal radius shortening, decrease the palmar and ulnar inclination angles, and cause uneven articular surface; these consequently cause secondary pain and joint dysfunction (Bartl et al., 2011). Plate fixation is increasingly being applied in the treatment of comminuted distal radius fractures, and short-term follow-up demonstrates satisfactory results (Huang et al., 2005). Moreover, the post-healing functional score after the application of palmar T-shaped locking plates is superior to those with other methods such as non-locking plate fixation (Osti et al., 2012), dorsal plate fixation (Rausch et al., 2013), and percutaneous Kirschner wire fixation (Grewal et al., 2011). Nevertheless, although the use of a palmar T-shaped locking plate combined with a dorsal plate (Ring et al., 2005) or the technique of combining volar plating with locked radial column plating or K-wire fixation (Tang et al., 2010) can achieve satisfactory results, they incur greater trauma and more late complications. In contrast, another study indicates that palmar T-plate fixation is superior to the external fixator with respect to the restoration of radial height and wrist function (Esposito et al., 2013).

Therefore, in the present study, the curative effects of the application of the external fixator combined with open reduction and palmar internal fixation with plates and simple application of the palmar internal fixation with plates on distal radius fracture were compared.

\section{MATERIAL AND METHODS}

\section{General information}

During the years from January 2004 to December 2011, all consecutive patients with distal radius fractures in our department of orthopaedics were retrospected. A total of 61 patients ( 27 males and 34 females) were included, all patients provided informed consent and this study received ethical approval from the institutional review board. Their mean age was 48.5 years (range: $25-68$ years old). The reasons for injuries included 29 cases of traffic acci- 
dents, 23 cases of tumbles, and 9 cases of falls from a height. Furthermore, 7 patients suffered from open fracture (all Gustillo type I or II), while the others suffered from closed fracture. All patients were classified as type $\mathrm{C}$ according to the $\mathrm{AO} / \mathrm{ASIF}$ classification, including 6,11 , and 44 cases of types $\mathrm{C} 1, \mathrm{C} 2$, and $\mathrm{C} 3$, respectively. Forty-two patients received simple application of internal fixation with palmar plates (plate group); among them, 3 received bone grafting. Meanwhile, 19 patients received external fixator combined with plate internal fixation (external fixator + plate group); none of them received bone grafting.

\section{Surgical methods}

\section{Simple palmar T-plate fixation}

A palmar Henry approach was used in the interval between the flexor carpi radialis tendon and radial artery. First, 2 screws were placed on the proximal part of the T-plate in order to support the distal bone blocks. Patients with severe bone defects received intraoperative iliac bone grafting.

\section{External fixator combined with palmar T-plate fixation}

Open fractures were initially thoroughly debrided. First, the external fixator was installed. Two small $\sim 0.5-\mathrm{cm}$ incisions were made around the dorsum of the second metacarpal bone. Two screws for the external fixator were placed in the radial dorsal incision as described above, $5 \mathrm{~cm}$ away from the fractured end. The wrist joint was fixed to the ulnar deviation of the palmar flexion by the external fixator. The fractured end was treated with preliminary manual reduction under a $\mathrm{C}$-arm fluoroscopic $\mathrm{X}$-ray machine. The palmar plate fixation was then performed as described above.

\section{Postoperative treatment and follow-up}

The plate group began initiative and passive functional exercises of the affected hand as well as isometric contraction and relaxation exercises of the shoulder and elbow in the immediate postoperative period. However, patients with obvious osteoporosis, serious fracture compression, or a large residual defect after fracture reduction required 3 weeks of postoperative plaster immobilization before functional exercise. The external fixator + plate group underwent follow-up X-rays every 3-4 weeks postoperatively. If the X-ray confirmed the fractures were well healed 6-10 weeks postoperatively, the external fixator was dismantled followed by the gradual introduction of functional exercises of the wrist and forearm. The affected wrist was examined for palmar flexion and dorsiflexion, ulnar and radial deviation, pronation, and supination 12 months postoperatively. The steps of the articular surface, palmar angle, ulnar deviation, and radial height (i.e., the length of radial styloid and ulnar styloid) were measured in the distal part of the affected radius.

\section{Evaluation standard}

All patients were followed up for 12 months. Wrist function was evaluated according to the improved McBride score and the wrist joint functional evaluation criteria of the 
New York Orthopedic Hospital (Fernandez and Jupiter, 1996). The comprehensive evaluation included pain and its influence on the patients' life and work, clinical inspection of wrist activities, grip strength, gross appearance, X-ray inspection of the reset of the articular surface, degree of radial shortening, recovery of the palmar and ulnar deviation angles, and osteoarthritis changes and complications. These aspects were graded as excellent, good, fair, or poor.

\section{Statistical analysis}

SPSS version 13.0 (SPSS Inc., Chicago, IL, USA) was used for statistical analysis. Data are reported as means \pm SD. Intergroup differences were evaluated by using the Student $t$-test. Indices presented as ranked data are reported as constituent ratios and analyzed by the $\chi^{2}$ test. The level of significance was set at $\mathrm{P}<0.05$.

\section{RESULTS}

\section{Treatment and follow-up results}

There were no significant differences between groups with respect to age or constituent ratio of fracture types $(\mathrm{P}>0.05$, Table 1$)$. All patients were followed up for 12 months postoperatively. X-rays showed all fractures healed and the articular surfaces were flat. The mean radial heights of the plate and external fixator + plate groups were $11.06 \pm 1.01$ and $13.53 \pm 1.20 \mathrm{~mm}$, respectively $(\mathrm{P}<0.05)$. The mean palmar angles in the plate and external fixator + plate groups were $7.41 \pm 2.76^{\circ}$ and $10.02 \pm 2.34^{\circ}$, respectively $(\mathrm{P}<0.05)$. Furthermore, the mean ulnar deviation angles in the plate and external fixator + plate groups were $19.56 \pm 6.87^{\circ}$ and $20.02 \pm 5.34^{\circ}$, which were not significantly different $(\mathrm{P}>0.05)$ (Figure $\left.1 \mathrm{~A}\right)$.

Regarding wrist function, $88.1 \%(37 / 42)$ and $89.5 \%$ (17/19) of patients in the plate and external fixator + plate groups had successful results (i.e., good or excellent), respectively $(\mathrm{P}>0.05$, Table 2). There were no significant differences in the range of activities including ulnar or radial deviation, pronation, or supination between groups $(\mathrm{P}>0.05)$. Furthermore, the palmar flexion and dorsiflexion were superior in the external fixator + plate group $(\mathrm{P}<$ 0.05 , Figure $1 \mathrm{~B})$. In the external fixator + plate group, there were no complications caused by the hypertraction of the external fixator, such as nonunion, finger stiffness, reflex sympathetic dystrophy, etc.; however, there was 1 case of superficial branching of the radial nerve injury, which was treated with relaxation of the external fixator and trophic nerve medication, ameliorating the feeling of numbness reported by the patient. In addition, there were 2 cases of pin tract infection, which improved as a result of enhanced skin care around the needle tract and use of antibiotics. Figure $1 \mathrm{C}$ and D was a typical case.

Table 1. Patient age and AO classification of fractures.
\begin{tabular}{lccccr}
\hline Group & $\mathrm{N}$ & Mean age (years) & & AO classification of fractures (N) \\
\cline { 3 - 6 } & & & $\mathrm{C} 1$ & $\mathrm{C} 2$ & $\mathrm{C} 3$ \\
\hline Simple plate group & 42 & 43.6 & 4 & 7 & 31 \\
External fixator + plate group & 19 & 51.8 & 2 & 4 & 13 \\
\hline
\end{tabular}




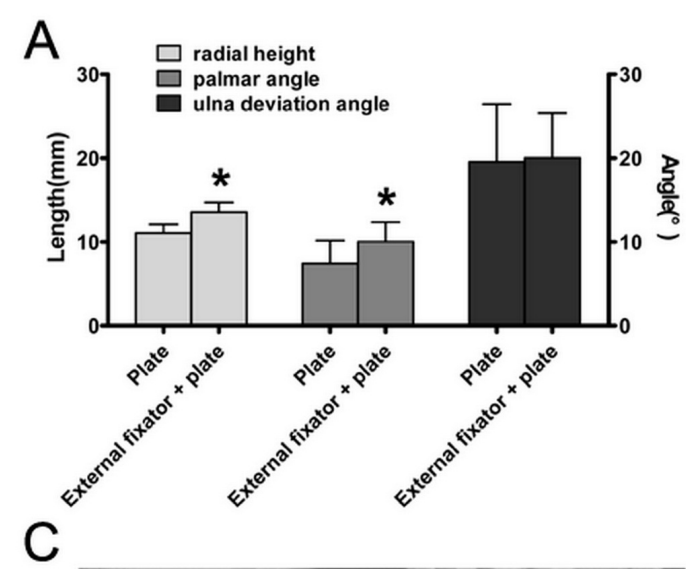

B
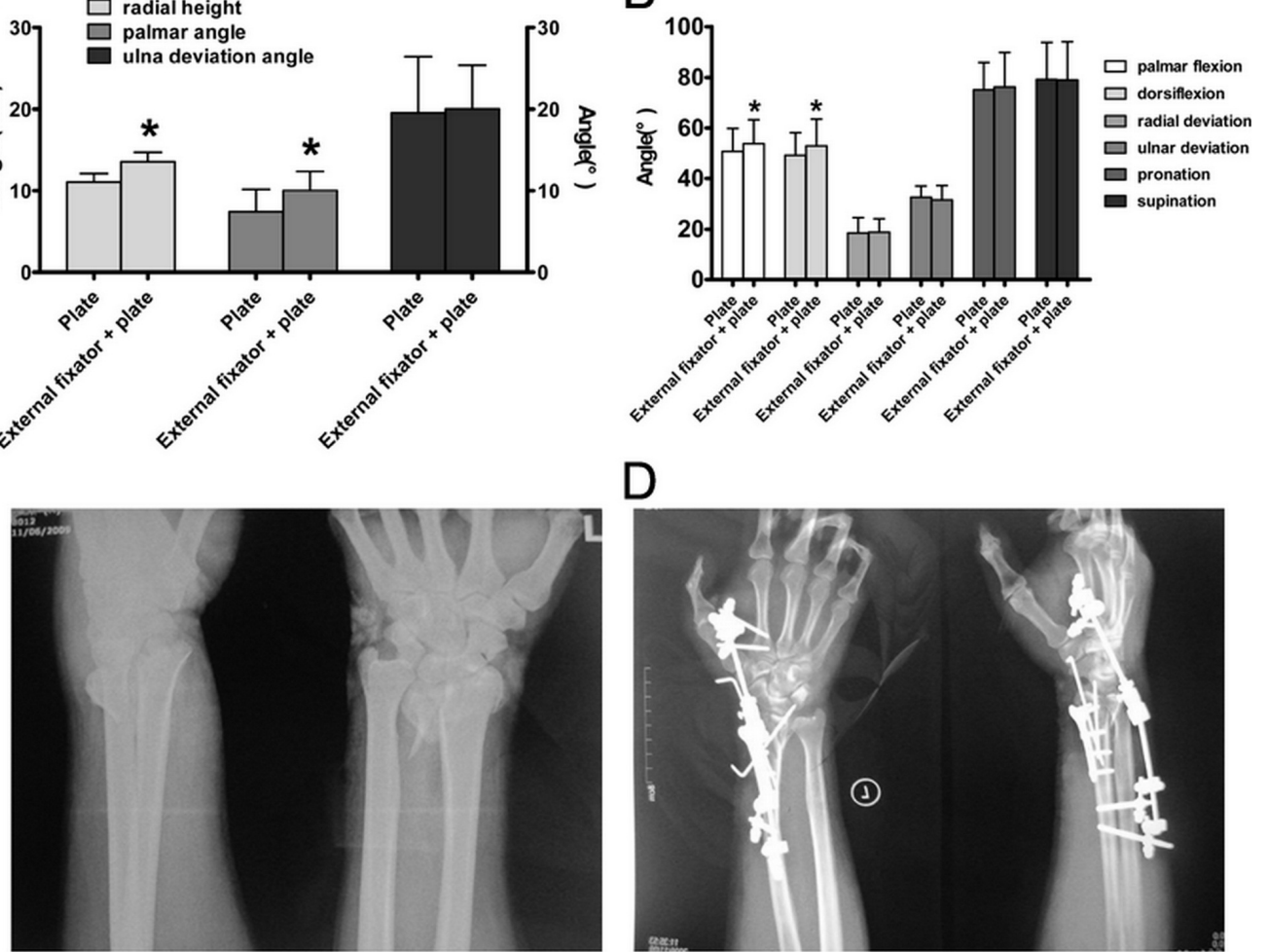

Figure 1. A. B. At the 12-month follow-up, patients who received external fixator combined with volar T-plate internal fixation had significantly greater radial length, radial tilt angle, and wrist flexion and extension compared than patients who received volar T-shaped plate internal fixation only. $* \mathrm{P}<0.05$. The volar tilt angle of the injured wrist, other ranges of movement, wrist joint function scoring, and the satisfactory result (i.e., good and excellent) rates did not differ significantly between groups $(\mathrm{P}>0.05)$. C. D. A patient, male, 45 years old, with a left distal radius comminuted fracture; C. preoperative anteroposterior and lateral views; D. postoperative anteroposterior and lateral views of internal fixation by external fixation combined with volar T-plate internal fixation.

Table 2. Wrist function scores 12 months postoperatively.

\begin{tabular}{lcccccc}
\hline Group & $\mathrm{N}$ & Excellent $(\mathrm{N})$ & Good $(\mathrm{N})$ & Fair $(\mathrm{N})$ & Poor $(\mathrm{N})$ & Success rate* $(\%)$ \\
\hline Simple plate group & 42 & 12 & 25 & 4 & 1 & $88.1 \%$ \\
External fixator + plate group & 19 & 6 & 11 & 1 & 1 & $89.5 \%$ \\
\hline
\end{tabular}

*The success rate was calculated as the sum of cases rated good and excellent divided by the total number of cases.

\section{DISCUSSION}

Comminuted distal radius fractures are unstable fractures caused by high-energy and direct axial compression injuries. Improper treatment can cause wrist joint degeneration, pain, stiffness, and other symptoms. Furthermore, obvious changes in the distal part of the articular surface of the radius, palmar and ulnar deviation angles, and radius shortening can severely restrict forearm rotation and wrist function (Azzopardi et al., 2005). In the present study, 19 
patients received an external fixator combined with a T-plate, while 42 received simple application of a T-plate. The results of the 12-month follow-up show the palmar flexion and dorsiflexion of the wrist, radial height, and palmar angle were significantly greater in the external fixator combined with the T-plate group than the simple T-plate group. However, there were no significant differences between groups with respect to radial-ulnar deviation, wrist range of motion (including pronation, supination, and radial and ulnar deviation), or wrist function score. Therefore, the effectiveness of the external fixator combined with T-plate internal fixation is an appropriate treatment for comminuted distal radius fractures. Most patients substantially recovered wrist, forearm, and hand function. Compared to the simple T-plate, the external fixator combined with T-plate internal fixation reduced the possibility of a postoperative re-shifting of fractured bones and kept the distraction of fractures to maintain radial height and prevent radial shortening, further evidencing its relative safety and effectiveness. The treatment of comminuted distal radius fractures should aim to maximize the recovery of the anatomical and mechanical structures (Kennedy and Hanel, 2013). Previous studies confirm that the normal wrist distal radius can withstand an axial load up to $80 \%$ (Short et al., 1987). If the deformed fractures in the distal part of the radius are healed, this kind of load transmission would be disordered, which would cause radiocarpal joint pain and decreased grip strength (Short et al., 1987).

Good reset of the articular surface is not only a key step in the treatment of distal radial intra-articular fractures, but is also important for achieving good treatment results. If the radial shortening exceeds $5 \mathrm{~mm}$, the articular surface shift exceeds $2 \mathrm{~mm}$, or the palmar angle tilts dorsally more than $20^{\circ}$, then the position and motion of the wrist joint would be altered; this would increase local stress by $27-51 \%$, which would cause a high incidence of traumatic osteoarthritis (Kilic et al., 2009). After fixation with gypsum and a splint, there was no strength to against the isometric contraction of muscle. Even if the early reset is satisfactory, there would still be a re-shift. Furthermore, the surgical incision could reset the articular surface and fracture blocks under direct visualization, so as to correct the wrist anatomy and restore its biomechanical function (Bales and Stern, 2012).

The use of plate fixation for the treatment of comminuted distal radius fracture is increasing; studies show that the postoperative recovery of the wrist joint is satisfactory (Chung et al., 2006; Arora et al., 2007). Pain experienced during joint motion is due to the unevenness of the joint; this can lead to decline in joint activities and is also the main cause of traumatic arthritis. Moreover, the palmar surgical approach can effectively repair the articular surface and firmly maintain the flat articular surface as a result of the plate. After reset, although the structure can be supported by the palmar plate, the repair of the articular surface will still be affected by the wrist stress, especially in elderly patients with osteoporosis and those with unstable comminuted fractures involving the articular surface due to violent injury (Day and Daly, 2012; Padegimas and Osei, 2013). For patients with serious bone defects, postoperative autologous or artificial bone grafting could effectively restore the radial length and prevent the postoperative collapse of the articular surface (Ozer et al., 2011; Gong et al., 2012; Hegde et al., 2013).

Radial shortening substantially reduces wrist activities and pain during activities, which is the main factor affecting wrist function. Motorized external fixators do not affect the stability of fractures during early postoperative wrist activities (Dienst et al., 1997). The external fixator can effectively maintain the radial length, but the longitudinal traction force parallel to the radius cannot restore the palmar angle of the articular surface in the distal part of the radius. Furthermore, simple application of the external fixator cannot stop subsequent dorsal distal collapse, which causes backward sloping of the articular surface. The results of 
the present study confirm that external fixator combined with application of the palmar plate can effectively restore the palmar angle and avoid radial collapse. Therefore, a simple external fixator is insufficient for comminuted distal radius fracture. Ruch et al. (2005) report that the application of a cross-wrist supporting plate in the treatment of serious comminuted distal radius fracture provides satisfactory clinical results with fewer complications. However, the cross-wrist supporting plate fixation also limits the early activities of the wrist, because the wrist joint must be fixed in this method; furthermore, the healing time is longer for comminuted fractures. Moreover, the articular surface cannot be adjusted according to the recovery of the patient to allow early functional exercises. In addition, this fixation requires the creation of a dorsal incision and breaking the fractured end for blood supply. Therefore, this fixation method is used less in clinical practice than the external fixator (Kennedy and Hanel, 2013).

Many studies report postoperative complications with the simple application of palmar plate fixation. However, improving the surgical technique and reducing the surgical waiting time could effectively avoid some early complications such as nerve dysfunction, infection, etc. (Ward et al., 2011). Other complications such as postoperative continuous wrist pains, tendon injuries, tenosynovitis, etc. (Alm-Paulsen et al., 2012; Kurimoto et al., 2012) did not occur in the present study. Other studies report that the hypertraction of the external fixator can cause nonunion, finger stiffness, reflex sympathetic dystrophy, and other complications; however, none of these occurred in the present study.

The comparison of function after the treatment of comminuted distal radius fracture showed both groups achieved satisfactory clinical results with a low incidence of postoperative traumatic osteoarthritis and high subjective patient satisfaction. Furthermore, the present statistical analysis showed that at the 12-month follow-up, there were no differences in the range of activities including pronation, supination, ulnar or radial deviation, or function scores between the 2 groups. In addition, the recovery of the palmar angle and radial length in the external fixator + plate group were superior to those of the plate only group, especially with respect to palmar flexion and dorsiflexion. These results indicate the external fixator is helpful for restoring and maintaining the radial length, effectively solving the significant decline of wrist joint activities and pain caused by radial shortening. However, a major limitation of this study is its small sample size. Therefore, the results remain to be confirmed by additional clinical studies.

\section{CONCLUSIONS}

An external fixator combined with T-plate internal fixation provides satisfactory treatment for comminuted distal radius fracture. Patients exhibited good recovery in wrist, forearm, and hand function. Compared to the simple T-plate, the external fixator combined with Tplate internal fixation reduced the possibility of the postoperative re-shifting of broken bones and kept the distraction of fractures to maintain radial height and prevent radial shortening. Therefore, it is a relatively safe and effective method for comminuted distal radius fractures.

\section{ACKNOWLEDGMENTS}

The authors thank Dr. Da-wei Wang for his help in the significant analysis and his support in obtaining ethical approval for this study. 


\section{REFERENCES}

Alm-Paulsen PS, Rod O, Rød K, Rajabi B, et al. (2012). Percutaneous pinning of fractures of the distal radius. J. Plast. Surg. Hand. Surg. 46: 195-199.

Al Khudairy A, Hirpara KM, Kelly IP and Quinlan JF (2013). Conservative treatment of the distal radius fracture using thermoplastic splint: pilot study results. Eur. J. Orthop. Surg. Traumatol. 23: 647-650.

Arora R, Lutz M, Hennerbichler A, Krappinger D, et al. (2007). Complications following internal fixation of unstable distal radius fracture with a palmar locking-plate. J. Orthop. Trauma 21: 316-322.

Azzopardi T, Ehrendorfer S, Coulton T and Abela M (2005). Unstable extra-articular fractures of the distal radius: a prospective, randomised study of immobilisation in a cast versus supplementary percutaneous pinning. J. Bone. Joint. Surg. Br. 87: 837-840.

Bales JG and Stern PJ (2012). Treatment strategies of distal radius fractures. Hand. Clin. 28: 177-184.

Bartl C, Stengel D, Bruckner T, Rossion I, et al. (2011). Open reduction and internal fixation versus casting for highly comminuted and intra-articular fractures of the distal radius (ORCHID): protocol for a randomized clinical multicenter trial. Trials 12: 84.

Chung KC, Watt AJ, Kotsis SV, Margaliot Z, et al. (2006). Treatment of unstable distal radial fractures with the volar locking plating system. J. Bone. Joint. Surg. Am. 88: 2687-2694.

Day CS and Daly MC (2012). Management of geriatric distal radius fractures. J. Hand. Surg. Am. 37: 2619-2622.

Dienst M, Wozasek GE and Seligson D (1997). Dynamic external fixation for distal radius fractures. Clin. Orthop. Relat. Res. 9: 160-171.

Esposito J, Schemitsch EH, Saccone M, Sternheim A, et al. (2013). External fixation versus open reduction with plate fixation for distal radius fractures: A meta-analysis of randomised controlled trials. Injury 44: 409-416.

Fernandez DL and Jupiter JB (1996). Fractures of the distal radius: a practical approach to management. Springer-Verlag, New York, 145-151.

Gong HS, Song CH, Lee YH, Rhee SH, et al. (2012). Early initiation of bisphosphonate does not affect healing and outcomes of volar plate fixation of osteoporotic distal radial fractures. J. Bone. Joint. Surg. Am. 94: 1729-1736.

Grewal R, MacDermid JC, King GJ and Faber KJ (2011). Open reduction internal fixation versus percutaneous pinning with external fixation of distal radius fractures: a prospective, randomized clinical trial. J. Hand. Surg. Am. 36: 1899-1906.

Hegde C, Shetty V, Wasnik S, Ahammed I, et al. (2013). Use of bone graft substitute in the treatment for distal radius fractures in elderly. Eur. J. Orthop. Surg. Traumatol. 23: 651-656.

Huang TL, Huang CK, Yu JK, Chiu FY, et al. (2005). Operative treatment of intra-articular distal radius fractures using the small AO external fixation device. J. Chin. Med. Assoc. 68: 474-478.

Kennedy SA and Hanel DP (2013). Complex distal radius fractures. Orthop. Clin. North. Am. 44: 81-92.

Kilic A, Ozkaya U, Kabukcuoglu Y, Sokucu S, et al. (2009). The results of non-surgical treatment for unstable distal radius fractures in elderly patients. Acta. Orthop. Traumatol. Turc. 43: 229-234.

Kurimoto S, Tatebe M, Shinohara T, Arai T, et al. (2012). Residual wrist pain after volar locking plate fixation of distal radius fractures. Acta. Orthop. Belg. 78: 603-610.

Osti M, Mittler C, Zinnecker R, Westreicher C, et al. (2012). Locking versus nonlocking palmar plate fixation of distal radius fractures. Orthopedics 35: e1613-1617.

Ozer K, Kilic A, Sabel A and Ipaktchi K (2011). The role of bone allografts in the treatment of angular malunions of the distal radius. J. Hand. Surg. Am. 36: 1804-1809.

Padegimas EM and Osei DA (2013). Evaluation and treatment of osteoporotic distal radius fracture in the elderly patient. Curr. Rev. Musculoskelet. Med. 6: 41-46.

Rausch S, Schlonski O, Klos K, Gras F, et al. (2013). Volar versus dorsal latest-generation variable-angle locking plates for the fixation of AO type 23C 2.1 distal radius fractures: A biomechanical study in cadavers. Injury 44: 523-526.

Ring D, Prommersberger K and Jupiter JB (2005). Combined dorsal and volar plate fixation of complex fractures of the distal part of the radius. J. Bone. Joint. Surg. Am. 87: 195-212.

Ruch DS, Ginn TA, Yang CC, Smith BP, et al. (2005). Use of a distraction plate for distal radial fractures with metaphyseal and diaphyseal comminution. J. Bone. Joint. Surg. Am. 87: 945-954.

Short WH, Palmer AK, Werner FW and Murphy DJ (1987). A biomechanical study of distal radial fractures. J. Hand. Surg. Am. 12: 529-534.

Tang P, Ding A and Uzumcugil A (2010). Radial column and volar plating (RCVP) for distal radius fractures with a radial styloid component or severe comminution. Tech. Hand Up. Extrem. Surg. 14: 143-149.

Ward CM, Kuhl TL and Adams BD (2011). Early complications of volar plating of distal radius fractures and their relationship to surgeon experience. Hand (N Y) 6: 185-189. 